\title{
The use of lithium in mixed states
}

\author{
Gabriele Sani, ${ }^{1,2 *}$ () and Andrea Fiorillo ${ }^{3}$
}

\author{
${ }^{1}$ NESMOS Department (Neuroscience, Mental Health, and Sensory Organs), School of Medicine and Psychology, Sant'Andrea Hospital, Sapienza \\ University, Rome, Italy \\ ${ }^{2}$ Centro Lucio Bini, Rome, Italy \\ ${ }^{3}$ Department of Psychiatry, University of Campania "L. Vanvitelli", Naples, Italy
}

Lithium is a unique drug. In more than 60 years of observation, it showed its multiple important clinical properties in treating mania, stabilizing mood alterations, preventing suicide and protecting from neurodegeneration. It was also the most extensively studied drug in psychiatry. Nevertheless, it is generally underprescribed. Specifically, lithium is virtually not considered in the treatment of patients affected by mixed affective states. Lithium is not suggested for the acute treatment of mixed affective states and is considered to be less effective than other mood stabilizers in the long-term management of these patients. The main reason why lithium has no indication for mixed states is the "lack of evidence." Actually, there are several reasons to consider lithium as an effective treatment in patients with mixed affective states.

Received 7 May 2019; Accepted 13 May 2019

Lithium is the most effective drug for the treatment of classic recurrent depressive and bipolar disorders. Moreover, lithium is considered the first-line treatment for acute mania or recurrent bipolar episodes. ${ }^{1}$

Surprisingly, lithium is not suggested for the acute treatment of mixed affective states, and is considered to be less effective than other mood stabilizers in the long-term management of patients with mixed episodes. The main reason why lithium has no indication for mixed states is the "lack of evidence," meaning lack of data from randomized controlled trials (RCTs). However, as we have learned from the long history of medicine and psychiatry, scientific advances do not exclusively come from randomized clinical trials. On a pros and cons basis, there are several reasons to consider lithium as an effective treatment in mixed states.

(1) Mixed states have been differently conceptualized during the last decades. Almost neglected in the first two editions of DSM system, DSM-III defined mixed states as episodes involving the "full symptomatic picture of both manic and major depressive episodes, intermixed or rapidly alternating every few days," DSM-IV required the simultaneous presence of a full manic and a full depressive episode, while DSM-5 requires a full mood episode with at least three non-overlapping symptoms. The clinical importance of specific symptoms over others has been

\footnotetext{
* Address correspondence to: Gabriele Sani, NeSMOS Department, Sapienza University, Via di Grottarossa 1035-1039, 00189, Roma, Italy. (Email: gabriele.sani@uniroma1.it)
}

underlined by several authors. ${ }^{2-4}$ In any case, all definitions recognize the highly unstable nature of mixed states and the presence of excitatory symptoms as clinical key features and main targets for any treatment. There is consensus over the common sense rule that the earlier the intervention, the better the outcome. It is not clinically meaningful to exclude lithium from first treatment options of these patients from the very beginning of the episodes. Precisely because they are very unstable, mixed states deserve to be treated with the most stabilizing treatment available. Moreover, lithium has a little risk to worsen the depressive component of a mixed episode compared to antipsychotics, and a little risk to exacerbate the excitatory one, compared to antidepressants. ${ }^{5}$ Someone could correctly point out that mixed states as well as rapid cyclicity respond less to lithium compared to classic bipolar patients. Promotion of alternatives to lithium has also led, in some cases, to unjustified implications that lithium has inferior efficacy to other alternaecially in these diagnostic subgroups. The point is that these patients are generally more resistant to all treatments, not only to lithium.

(2) Mixed states, especially mixed depressions, are associated with a dramatic risk of suicide attempts or completed suicide. ${ }^{6}$ Notably, mixed depression can be present not only in bipolar, but also in unipolar patients. Independently from diagnosis, suicide, often impulsive, is linked to the excitatory process that underlines mixed depression, or to the great psychomotor agitation and 
dysphoria inherent in mixed mania. Lithium is the only drug that showed a clear effect in preventing suicide independently from diagnosis. ${ }^{7}$ Although its antisuicidal mechanism is not well established, there is no rationale nor clinical reason to avoid prescribing lithium as soon as possible, since all other comparators are not as efficacious as lithium in preventing suicide. Nevertheless, in the real world, lithium is still strongly underprescribed, even in patients in whom it should be the first choice treatment, such as suicidal patients with BD. ${ }^{8}$

(3) Patients with mixed states are more likely to be younger at onset, to have more relapses, to spend longer time periods in illness, and to show a poorer outcome. Hence, clinicians should choose the best treatment option from the outset. Lithium is the most extensively studied medication in long-term follow-up studies over more than 60 years. Beyond its clinical effectiveness, studies showed that lithium's common side effects are less dangerous than the neurologic and metabolic ones associated with the long-term use of antipsychotics or other anticonvulsants, and that lithium does not increase the risk of mood destabilization, as antidepressants often do. ${ }^{9,10}$ Given its relative safety, lithium can be used in special populations where mixed states are common, such as pediatric and adolescent patients with bipolar disorder, where it can be prescribed from the age of 12 years; women with perinatal and postnatal depression, considering that the updated lithium-related teratogenic risk is quite low ${ }^{11}$; and elderly patients, in which lithium can be easily used along with other drugs. ${ }^{12}$

(4) People with mixed states are considered more likely to develop neurodegenerative changes, especially related to the increased number of episodes and to poor response to treatment. ${ }^{12}$ Gross brain structure is relatively preserved during the early phases of $\mathrm{BD}$, but alterations have been observed in chronic and more recurrent forms of the illness and progressive gray matter reduction has been associated with chronicity in people with BD-I. Recent evidence has pointed out the existence of a neuroprotective effect for lithium. The overall effect of lithium appears to be an increase or a "normalization" of gray matter volume, particularly in the hippocampus, the amygdala, the anterior and subgenual cingulate cortices. ${ }^{13}$ This effect is not shared with other mood stabilizers. Its putative neuroprotective properties render lithium the best candidate for the treatment of these patients.

(5) Often, RCTs are considered the "gold standard" of scientific research, while naturalistic studies are linked with lower levels of evidence. This is not always true. Naturalistic studies (effectiveness studies) are carried out under "real world" conditions, thus representing current daily clinical practice. ${ }^{14}$ While RCT data on lithium in mixed states are lacking, observational studies suggest that lithium could be an effective treatment for patients with mixed episodes (especially mixed depression). ${ }^{15}$

In the light of the above-mentioned considerations, we strongly believe that the role of lithium in the treatment of patients with mixed episodes should be reconsidered. According to the ancient aphorism "Primum, non nocere" (First, do not harm), Stahl ${ }^{16}$ invites to answer the question "Will lithium and mood stabilizers eventually be proven to have efficacy?" We think that answering this question is a priority need in the current clinical and pharmacological research in psychiatry. Instead of superficially considering that the available literature points to the exclusion of lithium from therapeutic options, we should rather call for more clinical research in this area. Considering that nearly all recent studies were industryfunded, and that there is currently no industrial interest to promote the use of lithium, this may lead to an underestimation of classical mood stabilizers, such as lithium. Hence, independent institutions, such as Public Agencies, Universities/Medical Schools or National or International Research Programs, should allocate funds to make these studies possible, preferably by taking into account patients' and relatives' perspectives. ${ }^{17,18}$

\section{Disclosures}

Gabriele Sani has received personal fees from Angelini, Lundbeck, Janssen.

Andrea Fiorillo has received personal fees from Lundbeck.

\section{REFERENCES:}

1. Fountoulakis KN, Gonda X, Baghai TC, et al. Report of the WPA section of pharmacopsychiatry on the relationship of antiepileptic drugs with suicidality in epilepsy. Int J Psychiatry Clin Pract. 2015; 19(3): 158-167.

2. Maj M, Pirozzi R, Magliano L, et al. Agitated depression in bipolar I disorder: prevalence, phenomenology, and outcome. Am J Psychiatry. 2003; 160(12): 2134-2140.

3. Koukopoulos A, Sani G. DSM-5 criteria for depression with mixed features: a farewell to mixed depression. Acta Psychiatr Scand. 2014; 129(1): 4-16.

4. Swann AC, Lafer B, Perugi G, et al. Bipolar mixed states: an international society for bipolar disorders task force report of symptom structure, course of illness, and diagnosis. Am J Psychiatry. $2013 ; 170(1): 31-42$.

5. Sani G, Perugi G, Tondo L. Treatment of bipolar disorder in a lifetime perspective: is lithium still the best choice? Clin Drug Investig. 2017; 37(8): 713-727.

6. Sani G, Tondo L, Koukopoulos A, et al. Suicide in a large population of former psychiatric inpatients. Psychiatry Clin Neurosci. 2011; 65(3): 286-295.

7. Baldessarini RJ, Tondo L. Suicide risk and treatments for patients with bipolar disorder. JAMA. 2003; 290(11): 1517-1519.

8. Bastiampillai T, Sharfstein SS, Allison S. Increasing the use of lithium and clozapine in US suicide prevention. JAMA Psychiatry. 2017; 74(4): 423.

9. Hayes JF, Marston L, Walters K, et al. Adverse renal, endocrine, hepatic, and metabolic events during maintenance mood stabilizer 
treatment for bipolar disorder: a population-based cohort study. PLoS Med. 2016; 13(8): e1002058.

10. Tondo L, Abramowicz M, Alda M, et al. Long-term lithium treatment in bipolar disorder: effects on glomerular filtration rate and other metabolic parameters. Int J Bipolar Disord. 2017; 5(1): 27.

11. Patorno E, Huybrechts KF, Bateman BT, et al. Lithium use in pregnancy and the risk of cardiac malformations. $N$ Engl J Med. 2017; 376(23): 2245-2254.

12. Muzina DJ. Pharmacologic treatment of rapid cycling and mixed states in bipolar disorder: an argument for the use of lithium. Bipolar Disord. 2009; 11(Suppl 2): 84-91.

13. McDonald C. Brain structural effects of psychopharmacological treatment in bipolar disorder. Curr Neuropharmacol. 2015; 13(4): $445-457$.
14. Fiorillo A, Luciano M, Sampogna G. Being influential or being misleading? Citation bias in psychiatric research and practice. Epidemiol Psychiatr Sci. 2018; 27(3): 242-243.

15. Sani G, Napoletano F, Vöhringer PA, et al. Mixed depression: clinical features and predictors of its onset associated with antidepressant use. Psychother Psychosom. 2014; 83(4): 213-221.

16. Stahl SM. Mixed-up about how to diagnose and treat mixed features in major depressive episodes. CNS Spectr. 2017; 22(2): 111-115.

17. Fiorillo A, Luciano M, Del Vecchio V, et al. Priorities for mental health research in Europe: A survey among national stakeholders' associations within the ROAMER project. World Psychiatry. 2013; 12(2): 165-170.

18. Wykes T, Haro JM, Belli SR, et al. Mental health research priorities for Europe. Lancet Psychiatry. 2015; 2(11): 1036-1042. 